\title{
A Retrospective Review to Evaluate the Demographics and Management Profile of Patients with Amyotrophic Lateral Sclerosis Attending a Multidisciplinary Clinic
}

\author{
Arash Jalali-Sohi, Leila Darki, and Said R Beydoun \\ Neuromuscular Division, Department of Neurology, Keck School of Medicine, University of Southern California, Los Angeles, CA, USA
}

DOI: https://doi.org/10.17925/USN.2020.16.2.110

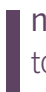
ntroduction: Amyotrophic lateral sclerosis (ALS) is a progressive neurodegenerative disease with heterogeneous age of onset, symptoms, topography, and progression. A multidisciplinary clinic (MDC) is the optimal form of healthcare delivery. We aimed to assess the demographic characteristics of, and the healthcare delivery to, patients with ALS attending an ALS MDC. Methods: A retrospective chart review of 82 patients attending our MDC was conducted. Results: Sixty percent of patients were male. Spinal-onset ALS was most common, had earlier onset, and occurred more in males. Median time from symptom onset to initial MDC visit was 18 months. More prevalent was the use of riluzole than edaravone. Percutaneous endoscopic gastrostomy (PEG) improved patient weight change $(p=0.026)$. Conclusions: Our patient cohort further confirms observed demographic trends. More should be done to identify barriers that delay referral to specialist services. Multidisciplinary care helps capture disease characteristics and progression. PEG placement reduces weight loss and should be pursued earlier. MDCs should characterize their patient populations to evaluate, and further optimize, healthcare delivery.

\section{Keywords}

Amyotrophic lateral sclerosis, multidisciplinary clinic, riluzole, edaravone, percutaneous endoscopic gastrostomy, glutamate,

superoxide dismutase 1, bulbar, spinal

Disclosures: Said R Beydoun has received research/grant support from Argenx, Catalyst, Mallinkrodt, Ra Pharma, and UCB; and has served as a consultant/speaker for Akcea, Alexion, Alnylam, CSL, Mitsubishi Tanabe, and Takeda. Arash Jalali-Sohi and Leila Darki have no financial or non-financial relationships or activities to declare in relation to this article.

Review Process: Double-blind peer review.

Compliance with Ethics: This study was a retrospective chart review and did not involve any studies with human or animal subjects performed by any of the authors.

Authorship: The named authors meet the International Committee of Medical Journal Editors (ICMJE) criteria for authorship of this manuscript, take responsibility for the integrity of the work as a whole, and have given final approval for the version to be published.

Access: This article is freely accessible at touchNEUROLOGY.com (c) Touch Medical Media 2020.

Received: May 7, 2020

Accepted: October 22, 2020

Published Online: December 23, 2020

Citation: US Neurology. 2020;16(2):110-5

Corresponding Author: Arash Jalali-Sohi,

Neuromuscular Division, Department of Neurology,

Keck Medicine of the University of Southern California

1520 San Pablo Street Suite 3000, Los Angeles,

CA 90033, USA. E: arashjalali96@gmail.com

Support: No funding was received in

the publication of this article.
Amyotrophic lateral sclerosis (ALS) is a progressive neurodegenerative disease that displays heterogeneous age of onset, symptoms, and progression. ${ }^{1,2}$ The disease presents clinically with upper and lower motor neuron features. ${ }^{3}$ There is no definitive test or assay that can be used to diagnose ALS, so the diagnosis is reached by ruling out other diseases that mimic the condition by utilizing clinical and electrodiagnostic findings. ${ }^{2}$ The Revised El Escorial criteria are widely used to diagnose and classify patients for research studies and clinical trials. ${ }^{4}$ Based on these criteria, patients can be classified as clinically definite, clinically probable, clinically probable (laboratory supported), and clinically possible. ${ }^{4}$

Only about $5-10 \%$ of ALS cases arise from inherited genetic mutation, while the vast majority of cases appear to originate sporadically. ${ }^{2}$ The most common genetic variants associated with ALS and frontotemporal dementia (FTD) are mutations in open reading frame 72 on chromosome 9 (C9orf72). ${ }^{5}$ This results in GGGGCC hexanucleotide repeats numbering in the hundreds to thousands, and may lead to a decrease in the expression of this gene's protein product. ${ }^{5}$ These repeat expansions in C9orf72 account for over $30 \%$ of familial cases and up to $10 \%$ of sporadic cases. ${ }^{.}$One study demonstrated that rare and potentially pathogenic variants in known ALS genes are present in over $25 \%$ of apparently sporadic cases and $64 \%$ of familial cases. ${ }^{7}$ This study also found that variations in more than one known ALS gene were present in a significant number of subjects, which influenced disease onset.? This lends support to the relevance of oligogenic inheritance in disease pathogenesis. ${ }^{7}$ Also present in sporadic cases, an additional $20 \%$ of familial ALS cases are caused by a toxic gain-of-function mutation in the gene SOD1, which codes for copper/zinc superoxide dismutase. ${ }^{1.46}$ This mutation causes increased free radical generation, as well as prion-like conformational changes in the protein that lead to the formation of disruptive intracellular aggregates. ${ }^{2.5}$ These intracellular aggregates inhibit normal proteosomic function, leading to disruptions in axonal transport and vital cellular functions. ${ }^{2}$

Besides the aforementioned mutations in C90rf72 and SOD1, there are several hypotheses for the underlying causes of, or contributors to, ALS. These include glutamate toxicity; increased reactive nitrogen and oxygen species and subsequent free radical damage; defects in RNA processing and endoplasmic reticulum stress and mitochondrial dysfunction; environmental toxins, viral infections; and insufficient availability of trophic factors. ${ }^{5}$ Glutamate excitotoxicity is implicated in the neuronal cell death that leads to ALS. ${ }^{4}$ Excessive glutamate-induced excitation of post-synaptic neurons can activate 
calcium-dependent enzymatic pathways that result in neurodegeneration. ${ }^{2}$ Furthermore, excessive glutamate excitation can lead to the formation of free radicals, which can cause irreparable cellular damage. ${ }^{2}$ The involvement of the immune system in ALS pathogenesis is an active area of investigation. ${ }^{5}$ Potential autoimmune activity has been reported in patients with ALS; however, there are no known distinctive immune markers in patients with ALS that clearly indicate immune-mediated motor neuron cell death and ALS pathogenesis. ${ }^{5}$ Currently, no definitive conclusions can be drawn regarding immune system involvement in ALS pathogenesis.

Most patients (approximately 70\%) present with spinal-onset ALS, where symptoms originate in the extremities. ${ }^{2,4}$ Patients with spinal-onset ALS experience fasciculations, weakness, spasticity, and muscle atrophy in their limbs. ${ }^{4}$ Bulbar-onset ALS is the second most common form and occurs in about $25 \%$ of patients. ${ }^{2}$ Bulbar symptoms of ALS include dysarthria, dysphagia, fasciculations and atrophy of the tongue, nasal speech, and sialorrhea. ${ }^{2}$ Bulbar symptoms pose significant risks to patients, such as increased likelihood of aspiration, which can lead to pneumonia. ${ }^{8}$ Dysphagia can lead to malnutrition, in which case a percutaneous endoscopic gastrostomy (PEG) may be recommended to help patients meet their nutritional needs. ${ }^{2}$ About $5 \%$ of patients present with respiratory-onset ALS, which is characterized by dyspnea, orthopnea, and morning headaches. ${ }^{2,9}$ As ALS progresses, many patients experience weakening of the diaphragm and accessory respiratory muscles. ${ }^{2}$ It is imperative that respiratory function be closely monitored, as respiratory failure is the main cause of death for patients with ALS. ${ }^{2}$ Respiratory dysfunction can be managed with non-invasive ventilation, which has been shown to improve quality of life (QOL) and survival. ${ }^{2}$ Invasive ventilation, delivered via tracheostomy, is an option when respiratory function declines such that non-invasive respiratory support becomes insufficient. ${ }^{2}$

A significant number of patients with ALS develop pseudobulbar affect.10 Patients with pseudobulbar affect experience episodes of uncontrollable and inappropriate laughing or crying, and this emotional lability can have negative social consequences. ${ }^{10}$ The Center for Neurologic Study-Lability Scale (CNS-LS) questionnaire is a tool used to objectively measure pseudobulbar affect. ${ }^{10}$ This questionnaire, which has been validated in patients with ALS, assesses a patient's ability to control laughter and crying..$^{10}$ The scoring ranges from seven, indicating no emotional lability, to 35 , which indicates severe emotional lability. ${ }^{10}$ A threshold score of 13 suggests the presence of pseudobulbar affect, as it corresponds to $82 \%$ of neurologists' clinical diagnoses in patients with ALS. ${ }^{10}$ Pseudobulbar affect is also associated with depression, as 30-35\% of patients with pseudobulbar affect are depressed. ${ }^{10}$ Depression is not uncommon in patients with ALS, as they may exhibit a decline in affect as they deal with the progressive nature of their condition. ${ }^{4}$

Patients diagnosed with ALS may also develop cognitive abnormalities, such as FTD. ${ }^{2,4}$ There is a known overlap between ALS and FTD, with 20-50\% of patients meeting the consensus criteria for probable or definite FTD. ${ }^{2} \mathrm{~A}$ comparison of magnetic resonance images between patients with ALS with and without FTD does show greater frontotemporal atrophy in patients with ALS and FTD. ${ }^{2}$ Patients who develop FTD may exhibit a decline in executive function that detrimentally affects their ability to conduct routine tasks. ${ }^{2} \mathrm{As}$ FTD continues to develop, patients can display impulsivity and problems with judgment. ${ }^{2}$ This can raise concerns in the context of patient treatment compliance and medical decision-making. ${ }^{2}$
Riluzole, a glutamatergic neurotransmission inhibitor, was the first drug approved by the United States Food and Drug Administration (FDA) for ALS treatment with modest benefits on survival. ${ }^{11}$ Studies have shown that riluzole can extend tracheostomy-free survival in patients with ALS by 2-3 months. ${ }^{11}$ In 2017, edaravone was approved by the FDA as the second ALS drug target to slow down progression. ${ }^{12}$ A Japanese study showed that, in a defined subgroup of patients, there was a significant reduction in ALS Functional Rating Scale-Revised (ALSFRS-R) decline in patients on edaravone when compared with patients on placebo after a 24-week period. ${ }^{12}$ This study design consisted of an enriched cohort, where steps were taken to manage variability in the patient population. ${ }^{6}$ Patients who progressed too slowly or too rapidly were excluded to ensure that the placebo and treatment groups had participants with similar rates of disease progression. ${ }^{6}$ This allowed for a more definitive assessment of drug efficacy. ${ }^{6}$ Concerns have been raised about the generalizability of the study results, especially given the selective nature of the inclusion criteria. ${ }^{13}$ In addition, the study provided no information regarding longer-term efficacy or survival. ${ }^{13}$ An open-label, 24-week extension phase of the study subsequently confirmed the safety and tolerability of edaravone, and suggested possible continued benefit. ${ }^{14}$ This study, however, was limited by the lack of a control population. ${ }^{14}$ Post-hoc analysis of data collected from the open-label extension study also provided evidence that edaravone may benefit patients with ALS for periods of treatment as long as 48 weeks. ${ }^{13}$

Dextromethorphan hydrobromide and quinidine sulfate (DMQ), sold under the brand name Nuedexta ${ }^{\circledR}$ (Anavir Pharmaceuticals, Inc., Aliso Viejo, CA, USA), is FDA approved for the treatment of pseudobulbar affect. ${ }^{10}$ In a study of 326 patients with ALS and multiple sclerosis with clinically significant pseudobulbar affect, the treatment group displayed significantly fewer episodes of laughing and crying when compared with the placebo group. ${ }^{10}$ A study by Smith et al. confirmed that this drug also improves bulbar function, specifically the enhancement of speech and swallowing, as well as the improved ability to handle oral secretions. ${ }^{15}$

Symptom management is the main form of healthcare delivery for patients with ALS. ${ }^{16}$ Due to this, multidisciplinary clinics (MDCS) have become an attractive means of managing patients with ALS. ${ }^{16}$ The MDC setting allows for patients to build valuable relationships with their care providers while promoting collaboration between specialties, which facilitates the delivery of quality care that is tailored to the specific needs of each patient. ${ }^{16-18}$ It is important that patients with ALS receive multidisciplinary care because MDC attendance has been shown to extend patient lifespan and improve QoL. ${ }^{16,18}$ Seeing patients consistently at an MDC over the course of their disease allows for measurements of their progression to be logged over time. This can help identify trends in the patient cohort and highlight areas of care that can be improved upon. MDCs offer the unique opportunity for healthcare providers to characterize their patient population.

We conducted an analysis of our patient cohort to compare its trends to those that exist in the literature in the hopes that such characterizations would highlight areas in which we can further improve care. By analyzing their MDC patient cohort, healthcare providers have the opportunity to assess their own healthcare delivery, thus allowing for improvements to patient care. This will help highlight unseen gaps in care and provide guidance for ways to optimize healthcare delivery in areas that are lacking. 


\section{Methods}

A retrospective chart review of new patients seen at the Keck Medicine of the University of Southern California ALS MDC from August 2017 to October 2019 was conducted. The study aimed to characterize the gender, age of onset, and spinal versus bulbar-onset ALS ratio to identify if our cohort was representative of the general ALS population, as described in the literature. It also aimed to evaluate the effectiveness of PEG placement; as well as the prevalence of FDA-approved therapy use, such as riluzole, edaravone, and DMQ; and to identify unseen gaps in care that can be improved upon. A Student's t-test was used to compare the difference in average age of symptom onset between spinal and bulbar patients. A paired t-test was used to compare the average percent change in weight per month for patients before and after receiving a PEG. The $p$-value used to denote statistical significance was 0.05 .

\section{Results}

Of the 82 patients seen in this MDC, 60\% ( $n=49)$ were male and $40 \%(n=33)$ were female. Spinal-onset ALS occurred the most frequently, appearing in $67 \%$ ( $n=55$ ) of patients. This was followed by bulbar-onset ALS, which was observed in $28 \%(n=23)$ of patients. Of the patient cohort, $5 \%(n=4)$ reported that they experienced spinal and bulbar symptoms simultaneously; all of these patients were male. No patients presented with respiratoryonset ALS. Patients presented to our clinic with a mean and median initial ALSFRS-R scores of 36 and 38, respectively, with a range from 19-46. The mean and median initial CNS-LS scores for our patient cohort were 12 and 11 , respectively. The initial CNS-LS scores in our cohort ranged from 7-26. Spinal-onset patients presented with an average initial CNS-LS score of 11, whereas bulbar-onset patients presented with an average initial CNS-LS score of 13. The difference between these two means, however, was not statistically significant. Median time from symptom onset to the first visit to our MDC was 18 months. Over the observed period, 10 patients expired. Six of these 10 patients had bulbar-onset ALS and the remaining four were spinal-onset.

Among patients with spinal-onset ALS, 69\% $(n=38)$ were male and 31\% $(n=17)$ were female. Conversely, an overwhelming majority of bulbar-onset patients were female. Of the 23 patients with bulbar-onset ALS, 70\% ( $n=16)$ were female and $30 \%(n=7)$ were male. For the entire patient cohort, the mean age of symptom onset was 60.2 (median 62), with a range of 31-89. There was a significant difference between the average age of onset of spinal and bulbar patients. Spinal-onset patients first experienced symptoms at an average age of 57.8 , compared with 65.7 for bulbar-onset patients $(p=0.017)$.

Edaravone was prescribed to $56 \%(n=46)$ of patients. Out of the 46 patients to whom edaravone was prescribed, $63 \%(n=29)$ initiated and maintained edaravone use and 24\% ( $n=11)$ never started infusions. The drug was initiated, but later discontinued in $13 \%(n=6)$ of patients for whom edaravone was prescribed. While the reasons for drug discontinuation varied, there were no reports stating that patients discontinued edaravone due to adverse drug reactions. Riluzole use was much more prevalent, with $73 \%(n=60)$ of the entire cohort taking the drug. Eighteen percent $(n=15)$ of patients were not taking riluzole and $9 \%(n=7)$ did not tolerate the drug and discontinued its use. The use of these drug therapies is not mutually exclusive, as $32 \%(n=26)$ of the cohort were using riluzole and edaravone simultaneously. Thirty-five percent $(n=29)$ of patients were taking DMQ, 62\% $(n=51)$ were not, and $2 \%(n=2)$ discontinued the drug. In follow-up visits, three patients expressed improvement in bulbar function after initiating DMQ.
The onset symptom profiles for patients using edaravone or riluzole closely matched the onset symptom profile of the patient cohort. For patients taking DMQ, however, 52\% $(n=15)$ had bulbar-onset ALS, 45\% ( $n=13)$ had spinal-onset, and $3 \%(n=1)$ experienced spinal and bulbar symptom onset simultaneously.

Patients presented with a mean forced vital capacity (FVC) of 77\% (median 79\%). Upon first presenting to our MDC, patient FVC ranged from 8-122\%. The patient with an FVC of $8 \%$ was noted as having difficulty creating a lip seal on the spirometer. Three patients were unable to have their FVC assessed due to the inability to create a lip seal on the spirometer. The prevalence of use for respiratory assistance systems was assessed. Non-invasive systems such as continuous positive airway pressure and bi-level positive airway pressure were used by $16 \%(n=13)$ of patients. Invasive respiratory assistance, as delivered through a tracheostomy, was in use by $7 \%(n=6)$ of patients.

In our patient cohort, 32\% $(n=26)$ of patients received a PEG recommendation. Of those 26 patients, $42 \%(n=11)$ had a PEG placed. Four of these 11 patients expired within the studied time period. Sixty-four percent $(n=7)$ of these 11 patients had bulbar-onset ALS and 36\% $(n=4)$ had spinal-onset ALS. A comparison was made between the average percent change in weight per month before and after PEG placement. Prior to PEG placement, patients had an average percent change in body weight per month of $-2.71 \%$. After having a PEG placed, the average percent change in weight improved significantly to $0.29 \%$ per month $(p=0.026$ ).

\section{Discussion}

It has been observed that men experience a greater incidence of this disease than women, with a male to female (M:F) gender ratio of 1.5:1.2.4 This was reflected in our patient cohort's M:F gender ratio, also 1.5:1, indicating a higher ALS incidence in men. The spinal and bulbar forms of ALS occur in different proportions, with occurrences of about $70 \%$ and $25 \%$, respectively. ${ }^{2}$ Our patient cohort mirrored these proportions closely. There is a notable difference in the gender distributions in our patient cohort regarding onset symptoms. The vast majority of patients with spinal-onset ALS were male. Despite our patient cohort being male-dominated, the bulbar-onset group had a much higher proportion of females than males. This difference is consistent with other studies; spinal-onset patients are predominantly male, whereas bulbar-onset patients are predominantly female. 2,4,19

Our patient cohort displayed a significant difference in the age of onset between spinal and bulbar patients, with spinal-onset patients being younger, on average, than bulbar-onset patients when they first experienced symptoms. Iwasaki et al. cites that their study experienced a similar result, with spinal-onset patients being diagnosed at a younger age than bulbar-onset patients. ${ }^{19}$ Our patient cohort had a mean age of symptom onset that falls well within the reported population range of 55-65 years. ${ }^{4}$ Demographically, our patient cohort is highly reflective of, and further confirms, trends that have been observed in the general ALS patient population. Six of the 10 patients who expired over the observed period had bulbar-onset ALS, despite the dominance of spinal-onset ALS within our cohort. This is indicative of the poorer prognosis that generally accompanies patients with bulbar symptoms, and further highlights the importance of multidisciplinary care. One study showed that patients with bulbar-onset ALS attending an MDC lived an average of 9.6 months longer 
than patients seen at general neurology clinics. ${ }^{16}$ Therefore, it is of vital importance that patients with bulbar symptoms are seen at an MDC as soon as they are diagnosed, as it can improve their symptom management and QoL, and extend their lifespan. ${ }^{20,21}$

One finding within our study that requires further attention is the time to first arrival to our MDC after symptom onset. After first experiencing symptoms, median arrival time to our MDC was 18 months. This is an area that should be improved upon. An Irish study of patients with ALS attending an MDC displayed a median time from symptom onset to MDC arrival of 14.6 months. ${ }^{21}$ This delay prevents patients from gaining access to resources and therapies that are shown to prolong survival and improve QoL. ${ }^{20}$ The care that patients receive in a multidisciplinary setting helps in the management of the many symptoms that patients with ALS experience, and in the prevention of further complications. In addition to being seen by neurologists, patients have sessions with speech, physical, and occupational therapists. These sessions help to provide patients with knowledge and techniques that allow them to better maintain independence and functionality, as well as manage symptoms that create hazards, such as falls and food aspiration. Multidisciplinary care also addresses the social aspects that arise with this disease. Patients and their families are seen by a social worker who helps ensure the patient is properly supported domestically and can provide access to support groups and foundations. Delayed referral to an MDC can be detrimental to a patient's prognosis, as the benefits of MDC attendance make it a prognostic factor of survival. .6,18 $^{1}$

A study of patients with ALS in Ireland found that patients attending an MDC lived, on average, 7.5 months longer than patients who attended general neurology clinics. ${ }^{16}$ Furthermore, a 2019 study of multidisciplinary ALS care in Spain compared patients attending an MDC with patients attending general neurology clinics, and found a 6-month increase in survival for patients attending an MDC. ${ }^{18}$ There is clearly a benefit seen by patients with ALS who attend an MDC as opposed to a general neurology clinic. To ensure that patients and their families receive the full potential of these benefits, it is imperative that newly diagnosed patients are referred to an MDC as soon as possible. For our own patients, this delay may be improved by raising awareness with general neurology clinics about our MDC.

Lack of awareness is not the only barrier that can prevent or prolong a patient from being seen in our MDC. The delay we observed between patients first experiencing symptoms and presenting to our MDC is due to multiple factors. Based on information available in patient charts, there are two major causes for this delay. The first is that some patients did not seek out medical attention upon the initial onset of symptoms. This may be due to the perceived harmlessness of their initial symptoms, such as minor spasticity and fasciculations. The second major contributing factor to this referral delay is a diagnostic delay once patients decide to seek out medical care. Patients who went to their primary care providers experienced a delay due to the fact that they needed to receive a referral to a neurologist. Since there is no definitive test for this condition, an ALS diagnosis is reached through a thorough process by which mimicking conditions are ruled out. ${ }^{2}$ This process, while necessary for a proper diagnosis, contributes to the delay we observed. Furthermore, it was noted that some patients opted to get a second opinion after they were initially diagnosed with ALS, and only gained a referral to our MDC after this process had concluded. Due to its retrospective nature, this study cannot assess the impacts of patient education level and socioeconomic status on referral time. This area should be investigated to further shed light on the possible barriers that prevent a timely diagnosis and MDC referral.

A less prevalent barrier, still worth noting, is that some patients were denied coverage for care at our MDC by their insurance providers. Insurance companies, while citing cost as a reason to deny a patient care at an MDC, may face greater expenses that can result from increased patient hospitalization that could have been prevented by multidisciplinary care.22 Furthermore, the costs of managing ALS through non-specialist services are equal to the costs of the specialized and optimized care provided by an MDC.22 Some patients would have been able to be seen sooner in our MDC if they had not had to wait through an appeal process to be granted access to our care. With the wealth of benefits that MDC attendance brings to a patient and their family, coverage denial can have a detrimental impact on a patient's prognosis, while possibly costing insurance companies more money over time. ${ }^{22}$ There needs to be a paradigm shift in how patients with ALS are managed. Earlier diagnosis and referral to specialist services gives patients the opportunity to enroll in clinical trials, as well as gain potential access to newly approved treatments and therapies as they become available. This ensures that patients are able to maximize their use of the resources available to them.

In a multidisciplinary setting, patients also develop strong relationships with their care team that improves clinicians' ability to address the unique needs of each patient. ${ }^{17,21}$ This setting also facilitates more effective communication between disciplines, which further improves the quality of care each patient receives..$^{18}$ This highly collaborative patient-centered approach to decision making contributes to increased patient satisfaction and improved health behaviors. ${ }^{23}$ Because of the nature of ALS and its progression, patients face a multitude of decisions that impact their symptom management and QoL.. ${ }^{23}$ Thanks to stronger patient-provider relationships and optimized interdisciplinary communication, each patient is able to participate in how their care plan is formulated. ${ }^{23}$ Healthcare providers come to understand the values and preferences of each patient and can formulate care plans that aim to preserve these values. ${ }^{23}$ This is especially important within the context of ALS because patients may experience declines in cognition and executive function, which negatively impacts their decision-making abilities. ${ }^{23}$ Despite this, multidisciplinary caregivers can still suggest healthcare decisions centered around the values and preferences of each individual patient. ${ }^{23}$ This understanding is important because patients and healthcare providers may differ on what they believe are effective courses of action. ${ }^{23}$

While multidisciplinary care is the optimal form of healthcare delivery for patients with ALS, it should be noted that it presents challenges in the context of emotional burden on the part of the healthcare provider.22 As a retrospective analysis, this study cannot draw conclusions about caregiver burden; however, it is an area that must be discussed when analyzing multidisciplinary care. It is known that healthcare professionals who provide care for patients with ALS experience stress and emotional burden that is directly related to their work. ${ }^{22}$ As previously stated, regular visits to an MDC fosters a closer relationship between patients and their care providers, which in turn improves healthcare delivery. ${ }^{17}$ While constructive to healthcare delivery, this stronger patient-provider relationship may increase stress for ALS care providers, who gain further emotional investment in their patients. ${ }^{22}$ Furthermore, the cognitive 
and behavioral changes that accompany ALS progression also increase caregiver burden and emotional stress. ${ }^{22,23}$ For this reason, there has been a push for support from healthcare organizations to ensure that ALS care providers have the resources to deal with these burdens. ${ }^{22}$ While an MDC is currently the most effective means of healthcare delivery for patients with ALS, it is still imperfect. Patient care is the first priority, but it is important that ALS care providers have access to professional mental and emotional support.22

Within our patient cohort, riluzole use was much more prevalent than edaravone use. There is clearly a discrepancy between these two drugs, both in terms of prescription rate and adherence to the prescription regimen. A significant contributor to this discrepancy may be the manner in which these drugs are taken. Riluzole is administered orally, taken as a 50 mg pill twice a day. ${ }^{24}$ With edaravone maintenance therapy, it is taken as a 60-minute intravenous infusion for 10 days during a 14-day period, followed by a 14-day drug-free period. ${ }^{12}$ The edaravone regimen is more demanding than that of riluzole, as it requires a time commitment on the part of the patient and/or the caregiver, specifically for patients who are receiving it at an infusion center. For our own patients who are receiving edaravone via home infusion, we found this to be a safe method that also circumvents the issues of visits to an infusion center. Due to incomplete information regarding exactly what type of infusion service each patient was using, we cannot make larger assessments on the impact that the infusion setting may have on edaravone adherence.

It is also important to have an objective discussion with patients before initiating any therapy, and to explain, in simple terms, the significance of trial results in the context of the study design, the importance of early treatment, and the limitations of any currently approved therapy as far as disease progression. If patients are not given the right information, it can raise false hope. Conversely, if they are only provided the limitations of the medication in a negative or discouraging way, they may be less eager to initiate or adhere to therapy. Patients may also ask about alternative therapies. It is important that care providers discuss the importance of using approved therapies with efficacies supported by scientific studies. Patients forgoing treatments that are known to be effective for unproven alternative therapies may experience earlier death, as therapies like riluzole and edaravone are shown to increase lifespan. ${ }^{4,11,12}$

DMQ is frequently prescribed for pseudobulbar affect..$^{15}$ More than half of the patients taking DMQ had bulbar-onset ALS, despite only $28 \%$ of our cohort being bulbar-onset. This indicates a possible association between bulbar-onset ALS and pseudobulbar affect, as patients with bulbar onset were more likely to be prescribed DMQ. This association is not unique, as Thakore et al. described a significant association between bulbar-onset ALS and the development of pseudobulbar affect. ${ }^{25}$ While primarily prescribed for pseudobulbar affect, DMQ has been shown to improve bulbar function in some patients. ${ }^{15}$ In our patient cohort, three patients specifically stated that they experienced noticeable improvements in bulbar function during follow-up visits after starting this medication. A crossover trial by Smith et al. demonstrated that patients taking DMQ experienced greater improvement in the bulbar domain of their ALSFRS-R score, compared with zero when they were on placebo. ${ }^{15}$ Why this improvement occurs is not fully understood, and further investigation into the mechanisms by which DMQ improves bulbar function is needed. This can hopefully lead to advancements in how bulbar symptoms are managed in patients with ALS, which can further improve their prognosis.
Many patients with ALS cannot meet their nutritional needs due to difficulties preparing and serving themselves food, or due to bulbar symptoms that make eating difficult. ${ }^{4}$ Furthermore, about $50-60 \%$ of patients with ALS are in a hypermetabolic state, which causes their caloric needs to increase. ${ }^{2}$ The combination of these factors can lead to malnutrition, weight loss, and further muscle atrophy. ${ }^{4}$ PEGs are recommended for patients experiencing these symptoms, even if dysphagia has yet to develop. ${ }^{2}$ Patients in our cohort who had a PEG placed showed a significant improvement in their percent change in body weight per month. This result further highlights the importance of PEG placement. Worsening nutritional status of patients with ALS is correlated to higher mortality, and a PEG can help counteract this..$^{20,26}$ Within our cohort, patients consistently declined or delayed suggestions for a PEG despite their worsening condition. A review of patient charts revealed that some patients delayed action for PEG insertion because they felt that their bulbar symptoms had not progressed enough to warrant this intervention. Hogden et al. assessed decision making among patients with ALS in a multidisciplinary setting and found that a common decision-making strategy used by patients was the "wait and see" approach. ${ }^{17}$ This mentality, cited as a mechanism for trying to maintain normalcy, can have negative impacts in the future. ${ }^{17}$ For PEG placement, we noted that some patients who were recommended to have this procedure waited until they were facing more serious bulbar dysfunction. This delay can increase the risk that comes with such a procedure. As ALS progresses, patients experience a decrease in FVC. ${ }^{9}$ Patients who undergo a procedure to have a PEG inserted experience greater morbidity as their FVC decreases. ${ }^{2}$ The decline in FVC that accompanies ALS progression means that when some patients finally decide to receive a PEG, they are facing heightened risks and a greater likelihood of experiencing complications during the procedure. $^{20}$ In patients for whom it is recommended, PEG placement should be pursued more strongly because of its potential to improve QoL and to counter the increased mortality that accompanies malnutrition. ${ }^{18}$

Patient efforts to preserve normalcy also extend into considerations for palliative care. Our multidisciplinary care team frequently have discussions with patients and their families regarding palliative care as a valuable resource. Despite these discussions, patient charts reveal that some patients denied recommendations for palliative care because they equate such care to "end-of-life" care. These patients, not believing their condition has progressed enough to require such care, deny themselves this useful resource. It is important that the benefits of palliative care are thoroughly explained, and that its false equivalency to being purely end-of-life care is addressed. Palliative care is perfectly suited for patients with ALS, as it is a practice that focuses on symptom management and medical decision-making for patients facing life-limiting illnesses. ${ }^{27}$ For cases where primary palliative care, as provided by the MDC, is insufficient, palliative care specialists can be extremely valuable in helping to manage the wide range of symptoms that patients with ALS experience. ${ }^{28}$ While palliative care is useful in the management of physical symptoms, such as pain, sialorrhea, and respiratory distress, it further offers benefits in the realm of psychological distress. ${ }^{28}$ Patients with ALS may experience depression, grief, and anxiety as they face the progressive nature of their condition. ${ }^{27}$ Furthermore, the decisions patients must make regarding their care, such as PEG placement and mechanical ventilation, can contribute to the psychological stress they experience. ${ }^{28}$ As experts in medical decision-making, symptom management, and support, palliative care specialists can add great value to the care profile of patients with ALS. ${ }^{28}$ To further improve the care our patients receive, it is essential that palliative 
care is more strongly pursued in patients for whom primary palliative care is insufficient. More constructive dialogue must be held such that the concerns and misconceptions patients may have regarding this valuable resource are properly addressed, as this specialized form of care has positive implications for patient QoL. ${ }^{28}$

\section{Conclusions}

The study of our cohort reaffirms gender and disease onset trends that have been previously observed within the ALS patient population. It also highlights areas that can be improved upon, namely MDC referral time and PEG placement. As stated, there is clearly a significant benefit for patients who are seen in an MDC.16,18,22 They experience increased lifespan and greater QoL than patients not seen in a multidisciplinary setting. ${ }^{16,18}$ Within our region, it is important that we raise further awareness about our MDC so that neurologists in our area can refer patients in a timelier manner. This will put patients in contact with the invaluable resources our MDC offers sooner, to ensure they can maximize the full range of resources available. There are no therapies available that improve or stabilize this condition, and existing medications are disease-modifying agents that can only slow the progression of the disease. ${ }^{6}$ These medications are shown to have greater benefit when initiated earlier in the disease course, so early diagnosis and referral is imperative. Healthcare providers must further address the coping strategies patients use to preserve normalcy, as these coping strategies can be detrimental to healthcare delivery and to patient QoL. Palliative care is a useful resource for patients with ALS, and it is important that care providers use their stronger patient-provider relationship to address patient concerns and misconceptions about this resource. Regarding increasing PEG placement, it is imperative that healthcare providers effectively circumvent the wait-and-see mentality. This may be done by educating patients about the dangers of this decision-making strategy in terms of FVC decline and increased procedure risk, as well as the association between worsening nutritional status and increased mortality. ${ }^{18,20}$ By studying our patient cohort, our multidisciplinary care team was able to find areas in which patient care can be further improved upon. It is important that other MDCs characterize their patient populations, so they may assess their own healthcare delivery to ensure that patients are truly receiving care that is optimized to meet their specific needs. The consolidation of resources makes MDCs the optimal method of healthcare delivery for patients with ALS, with clear improvements to symptom management, QoL, and lifespan extension. For these reasons, it is paramount that patients diagnosed with ALS are referred to an MDC in a timely manner. $\square$
1. Sabatelli $\mathrm{M}$, Conte $\mathrm{A}$, Zollino M. Clinical and genetic heterogeneity of amyotrophic lateral sclerosis. Clin Genet. 2013;83:408-16.

2. Kiernan MC, Vucic S, Cheah BC, et al. Amyotrophic lateral sclerosis. Lancet. 2011:377:942-55.

3. Sach M, Winkler G, Glauche V, et al. Diffusion tensor MRI of early upper motor neuron involvement in amyotrophic lateral sclerosis. Brain. 2004;127:340-50.

4. Wijesekera LC, Leigh PN. Amyotrophic lateral sclerosis. Orphanet J Rare Dis. 2009;4:3.

5. Lyon MS, Wosiski-Kuhn M, Gillespie R, et al. Inflammation, immunity, and amyotrophic lateral sclerosis: I. Etiology and pathology. Muscle Nerve. 2019;59:10-22.

6. Beydoun SR, Rosenfeld J. Edaravone in amyotrophic lateral sclerosis - Lessons from the clinical development program and the importance of a strategic clinical trial design. US Neurology. $2018 ; 14: 47-53$

7. Cady J, Allred P, Bali T, et al. Amyotrophic lateral sclerosis onset is influenced by the burden of rare variants in known amyotrophic lateral sclerosis genes. Ann Neurol. 2015;77:100-13.

8. Amin MR, Harris D, Cassel SG, et al. Sensory testing in the assessment of laryngeal sensation in patients with amyotrophic lateral sclerosis. Ann Otol Rhinol Laryngol. 2006;115:528-34.

9. Shoesmith $\mathrm{CL}$, Findlater K, Rowe A, et al. Prognosis of amyotrophic lateral sclerosis with respiratory onset. J Neurol Neurosurg Psychiatry. 2007;78:629-31.

10. Ahmed A, Simmons Z. Pseudobulbar affect: prevalence and management. Ther Clin Risk Manag. 2013:9:483-9.

11. Bellingham MC. A review of the neural mechanisms of action and clinical efficiency of riluzole in treating amyotrophic latera sclerosis: what have we learned in the last decade? CNS NeurosCi Ther. 2011;17:4-31.

12. Cruz MP. Edaravone (Radicava): a novel neuroprotective agent for the treatment of amyotrophic lateral sclerosis P T. 2018;43:25-8.

13. Shefner J, Heiman-Patterson T, Pioro EP, et al. Long-term edaravone efficacy in amyotrophic lateral sclerosis: post-hoc analyses of Study 19 (MCI186-19). Muscle Nerve. 2019;61:218-21.

14. Writing group on behalf of the edaravone (MCl-186) ALS 19 study group. Open-label 24-week extension study of edaravone (MCl-186) in amyotrophic lateral sclerosis. Amyotroph Lateral Scler Frontotemporal Degener. 2017:18:55-63.

15. Smith R, Pioro $E$, Myers $K$, et al. Enhanced bulbar function in amyotrophic lateral sclerosis: the Nuedexta treatment trial. Neurotherapeutics. 2017;14:762-72.

16. Traynor BJ, Alexander M, Corr B, et al. Effect of a multidisciplinary amyotrophic lateral sclerosis (ALS) clinic on ALS survival: a population based study, 1996-2000. J Neurol Neurosurg Psychiatry. 2003;74:1258-61.

17. Hogden $A$, Greenfield $D$, Nugus $P$, et al. What influences patient decision-making in amyotrophic lateral sclerosis multidisciplinary care? A study of patient perspectives. Patient Prefer Adherence. 2012:6:829-38.

18. Paipa AJ, Povedano MJ, Barcelo AJ, et al. Survival benefit of multidisciplinary care in amyotrophic lateral sclerosis in Spain: association with noninvasive mechanical ventilation. J Multidiscip Healthc. 2019;12:465-70.

19. Imasaki Y, Ikeda K, Ichikawa $Y$, et al. The diagnostic interval in amyotrophic lateral sclerosis. Clin Neurol Neurosurg. 2002;104:87-9.

20. Zarei S, Carr K, Reiley L, et al. A comprehensive review of amyotrophic lateral sclerosis. Surg Neurol Int. 2015;6:171.

21. Galvin M Madden C Maguire S, et al. Patient journey to a specialist amyotrophic lateral sclerosis multidisciplinary clinic: an exploratory study. BMC Health Serv Res. 2015;15:571.

22. Hogden A, Foley G, Henderson RD, et al. Amyotrophic lateral sclerosis: improving care with a multidisciplinary approach. J Multidiscip Healthc. 2017;10:205-15.

23. Hogden A, Greenfield D, Nugus P, et al. Development of a model to guide decision making in amyotrophic lateral sclerosis multidisciplinary care. Health Expect. 2015;18:1769-82.

24. Groeneveld GJ, van Kan HJM, Lie-A-Huen L, et al. An association study of riluzole serum concentration and survival and disease progression in patients with ALS. Clin Pharmacol Ther. 2008;83:718-22.

25. Thakore NJ, Pioro EP. Laughter, crying and sadness in ALS. J Neuro Neurosurg Psychiatry. 2017;88:825-31.

26. Marin B, Desport JC, Kajeu P, et al. Alteration of nutritional status at diagnosis is a prognostic factor for survival of amyotrophic lateral sclerosis patients. J Neurol Neurosurg Psychiatry. 2011;82:628-34

27. Blackhall $\sqcup$. Amyotrophic lateral sclerosis and palliative care: where we are, and the road ahead. Muscle Nerve. 2012;45:311-8

28. Brizzi K, Paganoni S, Zehm A, et al. Integration of a palliative care specialist in an amyotrophic lateral sclerosis clinic: observations from one center. Muscle Nerve. 2019;60:137-40. 